\title{
First-principles approach to monitoring the band gap and magnetic state of a graphene nanoribbon via its vacancies
}

\author{
M. Topsakal, ${ }^{1}$ E. Aktürk, ${ }^{1}$ H. Sevinçli, ${ }^{1,2}$ and S. Ciraci ${ }^{1,2, *}$ \\ ${ }^{1}$ UNAM-Institute of Materials Science and Nanotechnology, Bilkent University, Ankara 06800, Turkey \\ ${ }^{2}$ Department of Physics, Bilkent University, Ankara 06800, Turkey \\ (Received 7 August 2008; revised manuscript received 2 December 2008; published 30 December 2008)
}

\begin{abstract}
Using first-principles plane-wave calculations we predict that electronic and magnetic properties of graphene nanoribbons can be modified by the defect-induced itinerant states. Structure optimization gives rise to significant reconstruction of atomic structure, which is in good agreement with transmission electron microscope images. The band gaps of armchair nanoribbons can be modified by hydrogen-saturated holes. The band-gap changes depend on the width of the ribbon as well as on the position of the hole relative to the edges of the ribbon. Defects due to periodically repeating vacancy or divacancies induce metallization as well as magnetization in nonmagnetic semiconducting nanoribbons due to the spin polarization of local defect states. Antiferromagnetic ground state of semiconducting zigzag ribbons can change to ferrimagnetic state upon creation of vacancy defects, which reconstruct and interact with edge states. Even more remarkable is that all these effects of vacancy defects are found to depend on their geometry and position relative to the edges. It is shown that these effects can, in fact, be realized without really creating defects.
\end{abstract}

DOI: 10.1103/PhysRevB.78.235435

PACS number(s): 73.22.-f, 74.62.Dh, 75.70.Ak, 75.75.+a

\section{INTRODUCTION}

Its unusual electronic energy-band structure and charge carriers resembling massless Dirac fermions have made graphene honeycomb structure an active field of research. ${ }^{1-5}$ Quasi-one-dimensional graphene ribbons have even more interesting electronic and magnetic properties depending on their size and edge shape ${ }^{6-13}$ Edge states of zigzag ribbons with opposite spin polarization ${ }^{6}$ and band gaps varying with the width of the ribbons have been of particular interest. ${ }^{11}$

Theoretical studies have predicted that energetic electrons and ions can induce polymorphic atomic defects, such as vacancies in graphene. ${ }^{14}$ Using high-resolution transmission electron microscopy (TEM) techniques, the observation of vacancies has been reported. ${ }^{15}$ Recent studies have shown that vacancies created on two-dimensional (2D) graphene by high-energy electron or ion irradiation can induce magnetism in a system consisting of only $s p$ electrons. ${ }^{16,17}$ It has been argued that Stoner magnetism with high $T_{C}$ originates from the spin-polarized extended states induced by the vacancy defects, while Ruderman-Kittel-Kasuya-Yoshida (RKKY) coupling is suppressed. Brey et al. ${ }^{18}$ argued that the RKKY interaction is primarily ferromagnetic for moments on equivalent sublattices but becomes antiferromagnetic (AFM) on opposite sublattices. On the other hand, it is predicted that the RKKY interaction between the local moments induced by cracks and large voids is ferromagnetic. ${ }^{19}$ These effects of defects on one-dimensional (1D) semiconducting graphene nanoribbons should be more complex and interesting because their band gap, magnetic state, and symmetry are expected to intervene. Calculations based on the mean-field Hubbard model by Palacios et al. ${ }^{20}$ have revealed interesting electronic and magnetic properties of single vacancy and voids in armchair nanoribbons. Transport calculations by means of Landauer approach within the tight-binding model showed that the variation in conductance with chemical potential strongly depends on the number of removed sublattice sites. ${ }^{9}$
Present study based on extensive first-principles as well as empirical tight-binding (ETB) calculations has shown that the band gap and magnetic state of any armchair or zigzag nanoribbons can be modified by single or multiple vacancies (holes). The effects of these defects depend on their symmetry, repeating periodicity, and positions relative to the edges of the ribbon. Our results indicate significant interaction between magnetic edge states of zigzag graphene ribbons and localized spin states of vacancy defects. At the defect site the atomic structure undergoes a reconstruction which is almost identical with the transmission electron microscopy images reported earlier. ${ }^{15}$ Even if the formation of periodic defects of desired symmetry may not be easily achieved, similar effects can be created through the potential difference across the ribbon applied by periodically arranged tips. When combined with various properties of nanoribbons, these results can initiate a number of interesting applications. We believe that our results are important for further studies, since the graphene ribbons can now be produced with precision having widths below $10 \mathrm{~nm}$, and nanodevices can be fabricated thereof. ${ }^{21,22}$

\section{MODEL AND METHODOLOGY}

We have performed first-principles plane-wave calculations within density-functional theory (DFT) using projector augmented wave (PAW) potentials. ${ }^{23}$ The exchangecorrelation potential has been approximated by generalized gradient approximation (GGA) using PW91 (Ref. 24) functional both for spin-polarized and spin-unpolarized cases. All structures have been treated within supercell geometry using the periodic boundary conditions. A plane-wave basis set with kinetic-energy cutoff of $500 \mathrm{eV}$ has been used. In the self-consistent potential and total-energy calculations the Brillouin zone $(\mathrm{BZ})$ is sampled by $(1 \times 1 \times 35)$ special $\mathbf{k}$ points for ribbons. This sampling is scaled according to the size of superlattices. All atomic positions and lattice con- 
(a)

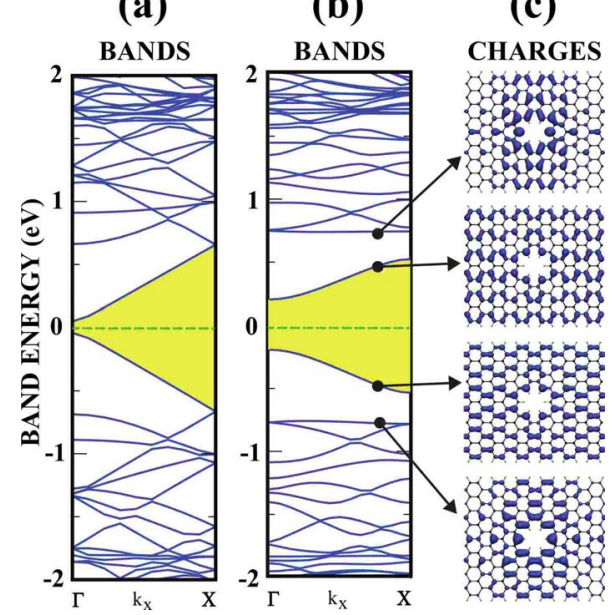

FIG. 1. (Color online) (a) Energy-band structures of $\operatorname{AGNR}(N=34 ; l=6)$ with and (b) without a hole consisting of six carbon vacancies. (c) Charge-density isosurfaces of selected states. Carbon atoms (represented by black circles), which have coordination number lower than three, are terminated by hydrogen atoms (represented by small gray circles).

stants are optimized by using the conjugate gradient method, where the total energy and atomic forces are minimized. The convergence for energy is chosen as $10^{-5} \mathrm{eV}$ between two steps, and the maximum force allowed on each atom is less than $0.02 \mathrm{eV} / \AA$. Numerical plane-wave calculations have been performed by using VASP package. ${ }^{25,26}$

\section{EFFECTS OF VACANCY DEFECTS ON GRAPHENE RIBBONS}

We start by summarizing the electronic properties of graphene nanoribbons which are relevant for the present study. We will consider hydrogen-terminated nanoribbons if it is not stated otherwise. Armchair graphene nanoribbons, $\operatorname{AGNR}(N)$ (with $N$ being the number of carbon atoms in the primitive unit cell), are nonmagnetic semiconductors. The band gap, ${ }^{12,27} E_{G}$, of a bare or hydrogen-terminated $\operatorname{AGNR}(N)$ is small for $N=6 m-2$ (with $m$ being an integer), but from $N=6 m$ to $N=6 m+2$ it increases, and passing through a maximum it again becomes small at the next minimum corresponding to $N=6 m+4$. As $E_{G}$ oscillates with $N$ its value shall decrease eventually to zero as $N \rightarrow \infty$. Bare and hydrogen-terminated zigzag graphene nanoribbons, ZGNR $(N)$, are also semiconductors with $E_{G}$ decreasing consistently as $N$ increases for $N>8$, but their edge states give rise to AFM ground state. ${ }^{6}$ We will show that these magnetic and electronic properties can be modified by defects originating from vacancies or holes created in those ribbons.

\section{A. Armchair nanoribbons}

A typical hole is created by removing carbon atoms at the corners of any hexagon of an armchair ribbon and subsequently by terminating the remaining six twofoldcoordinated carbon atoms with hydrogen atoms. Figures 1(a) and $1(\mathrm{~b})$ show that the electronic structure of $\operatorname{AGNR}(34)$ is (a)

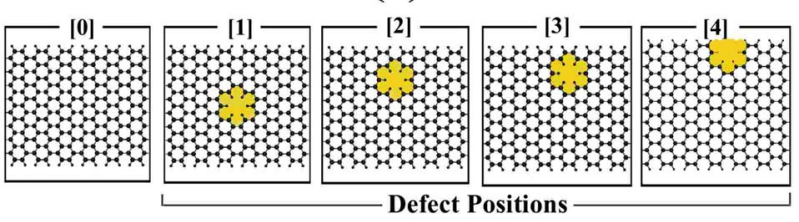

(b)

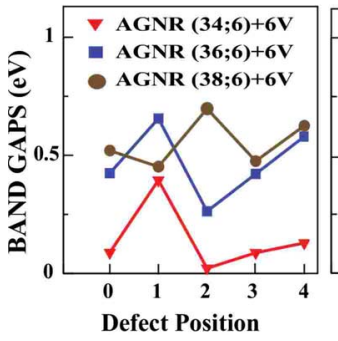

(c)

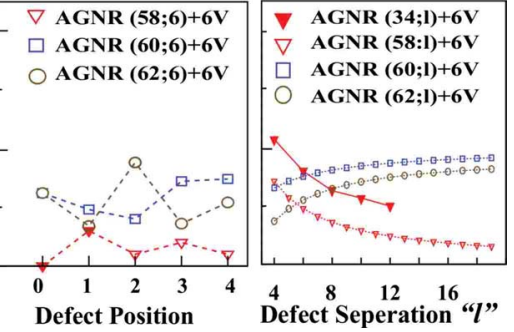

FIG. 2. (Color online) Effect of a periodic hole on the electronic structure of armchair nanoribbon. (a) Positions of a hole in the ribbon are indicated by numerals. The nanoribbon without a hole is specified by "0." Carbon atoms (black circles), which have coordination number lower than three, are terminated by hydrogen atoms (represented by small gray circles). [(b) and (c)] Variations in band gaps of $\operatorname{AGNR}(N, l)$ with the position of the hole specified as $6 \mathrm{~V}$. (d) Variation in the band gap with repeat periodicity, $l$. Results for $N<58$ are obtained by first-principles calculations.

strongly modified by such a hole which is placed at the center of the ribbon. The hole repeats itself at each supercell which comprises six primitive cells corresponding to a repeat period of $l=6$. This ribbon having a periodic hole (or defect) is specified as $\operatorname{AGNR}(N ; l)$ and has a nonmagnetic ground state. Despite large separation of periodic defect which hinders their direct coupling, such a strong modification of the band gap is somehow unexpected. However, it is an indirect effect and it occurs since the itinerant (Bloch) states of band edges are modified by the defect. At the end, the direct band gapp $^{27}$ at the $\Gamma$ point has widened from 0.09 to $0.40 \mathrm{eV}$ due to a defect situated at the center of the ribbon. AGNR(36) exhibits the similar behavior; namely, its band gap increases when a similar hole repeats itself at each supercell consisting of six primitive cells. In contrast, the band gap of $\operatorname{AGNR}(38)$, which is normally larger than that of $\operatorname{AGNR}(34)$, is reduced if the same hole is introduced at its center. In addition, states localized around the defect have formed flat bands near the edge of valence and conduction bands because of their reduced coupling. These results are in agreement with previous works revealing states localized at the vacancy defect. ${ }^{9}, 19,28,29$

Even more interesting is that the above effects of the hole consisting of six carbon vacancies are strongly dependent on its position relative to both edges of the nanoribbon as depicted in Fig. 2(a). As shown in Fig. 2(b), the changes in band gap depend on $N$ of AGNR (in the family specified as $6 m+q$, with $q$ being $-2,0$, and +2$)$ as well as on the position of the hole. We note that the variation in the band gap with the position of the hole relative to the edges of the ribbon shows similar trends for both $\operatorname{AGNR}(34 ; 6)$ and $\operatorname{AGNR}(36 ; 6)$. 
However, AGNR $(38 ; 6)$ displays different trends.

ETB calculations indicate that a similar behavior is also obtained in $\operatorname{AGNR}(N ; l)$ 's having relatively larger $N$ (i.e., $N=58,60,62)$ in Fig. 2(c). As expected the effect of the hole on the band gap depends on the repeat periodicity. As shown in Fig. 2(d), the effect of defect decreases with increasing repeat periodicity $l$. We found that larger holes with different geometry and rotation symmetry can result in diverse electronic structure and confined states. It should be noted that a repeating hole can also modify the mechanical properties. For example, the stiffness of a ribbon is reduced by the presence of a hole. The force constant, $\kappa=\partial^{2} E_{T} / \partial c^{2}$ (with $E_{T}$ being the total energy and $c$ being the lattice constant), calculated for $\operatorname{AGNR}(34 ; 6)$ with a hole at its center $(\kappa$ $\left.=6.03 \mathrm{eV} / \AA^{2}\right)$ is found to be smaller than that without a hole $\left(\kappa=7.50 \mathrm{eV} / \AA^{2}\right)$.

We now show that different types of vacancies in the same armchair nanoribbon give rise to different changes in electronic and magnetic properties. A divacancy created in $\operatorname{AGNR}(22)$ can cause a dramatic change in the electronic state of the ribbon when it is repeated with the periodicity of $l=5$. Such an armchair ribbon is specified as $\operatorname{AGNR}(22 ; 5)$. The divacancy first relaxes and forms an eightfold ring of carbon atoms which is adjacent to six hexagons and two pentagons. In Fig. 3(a) we see that the nonmagnetic and semiconducting $\operatorname{AGNR}(22)$ with a band gap of $E_{G}$ $=0.18 \mathrm{eV}$ becomes a nonmagnetic metal, since a flat band derived from the defect occurs below the top of the valance band edge and causes a metallic state. The states of this band have charge localized at the atoms surrounding the defect. Calculations performed with different repeat periods $l$ $=3,4,5,10$ show that the dispersion of this band decreases with increasing $l$. The structure including a single divacancy, which has two sublattices with equal number of carbon atoms, $N_{A}$ and $N_{B}$, remains bipartite even after relaxation. At the end, the net magnetic moment per unit cell is $\mu=\left(N_{A}\right.$ $\left.-N_{B}\right) \mu_{B}=0$.

The effect of a single carbon vacancy becomes even more interesting. ${ }^{30}$ A single vacancy created in $\operatorname{AGNR}(22)$ is relaxed and the threefold rotation symmetry is broken due to Jahn-Teller distortion. At the end, a nine-sided ring forms adjacent to a pentagon as shown in Fig. 3(b). All carbon atoms on this ring have threefold coordination except for the single one that remains twofold coordinated with a $s p^{2}$-dangling bond. Owing to the spin polarization of this $s p^{2}$-dangling bond and adjacent orbitals at the defect site, the system obtains an unbalanced spin. The difference of total charge density corresponding to different spin states, i.e., $\Delta \rho_{T}=\rho_{T}^{\uparrow}-\rho_{T}^{\downarrow}$ is nonzero and exhibits a distribution shown in Fig. 3(b). Because of unbalanced spin, $\operatorname{AGNR}(22 ; 5)$, which was nonmagnetic when perfect, gains a net magnetic moment of $\mu=1 \mu_{B}$ per unit cell. We note that this calculated net magnetic moment is in compliance with Lieb's theorem ${ }^{31}$ since $N_{A}-N_{B}=1$. The difference charge density, $\Delta \rho$, is calculated for the spin-down and spin-up bands just below the Fermi level and the lower-lying spin-up band at $\sim-0.5 \mathrm{eV}$. The former two bands are slight spin split and are formed from the combination of $\pi$ orbitals of carbon atoms surrounding the vacancy. The lower-lying spin-up band is derived mainly from the $s p^{2}$-dangling bond orbital of twofold- (a)
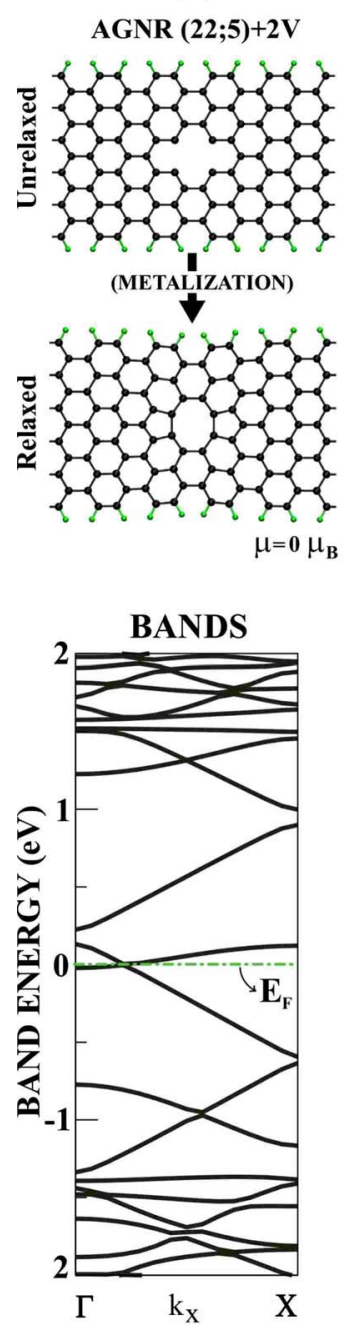

(b)

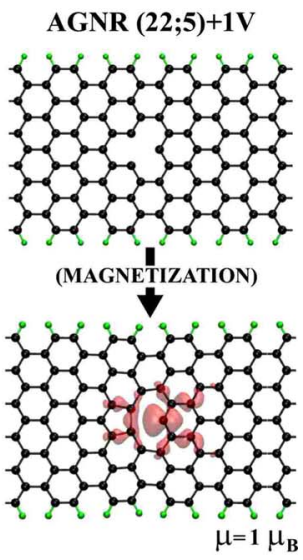

BANDS

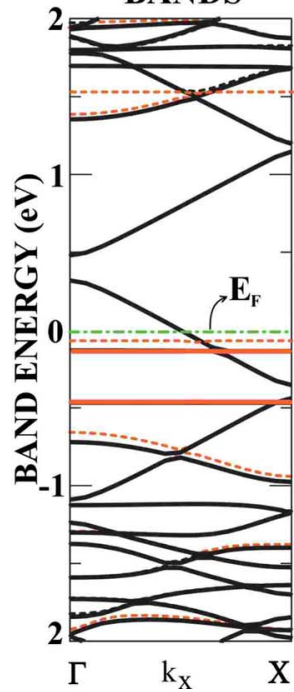

FIG. 3. (Color online) (a) Metallization of the semiconducting $\operatorname{AGNR}(22)$ by the formation of divacancies with a repeat period of $l=5$. (b) Magnetization of the nonmagnetic $\operatorname{AGNR}(22)$ by a defect due to the single carbon atom vacancy with the same repeat periodicity. Isosurfaces around the vacancy corresponding to the difference of the total charge density of different spin directions, $\Delta \rho_{T}$, indicate a net magnetic moment. Solid (red) and dashed (red) lines are for spin-up and spin-down bands; solid (black) lines are nonmagnetic bands.

coordinated carbon atom. The dispersive nonmagnetic band at the edge of the valence band becomes partially emptied since its electrons are transferred to the flat spin bands below. Eventually, the semiconducting ribbon becomes metallic.

The recent work by Palacios et al. ${ }^{18}$ based on the meanfield Hubbard model presented an extensive discussion on the effects of vacancy defects on graphene and graphene nanoribbons but only on the armchair ones. While they consider various vacancy defects, only the single vacancy is common between their work and the present work. In spite of the fact that they ignored the lattice distortion and assumed the same on-site energy for the edge, as well as for the bulk carbon atoms, our results are in overall agreement with theirs. In particular, the magnetic properties induced by the vacancy defects comply with Lieb's theorem ${ }^{31}$ in both 
(a)

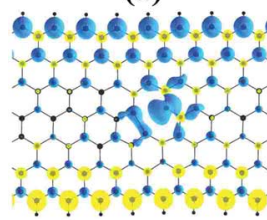

$\mathbf{E}_{\mathrm{T}}=-\mathbf{1 0 6 9 . 1 3} \mathrm{eV}, \boldsymbol{\mu}=1.94 \mu_{\mathrm{B}}$ $\mathrm{E}_{\mathrm{G}}^{\dagger}=0.29 \mathrm{eV}, \mathrm{E}_{\mathrm{G}}^{\downarrow}=0.06 \mathrm{eV}$

(d)

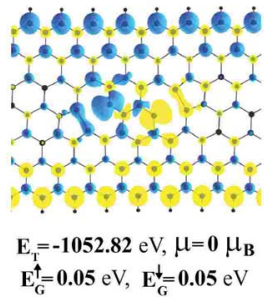

(b)

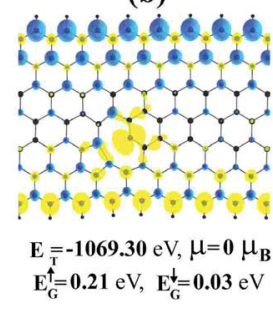

(e)

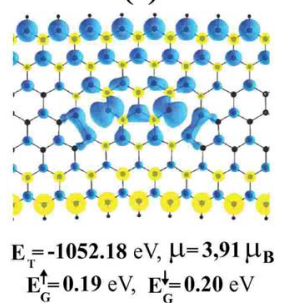

(c)

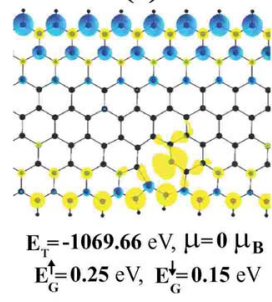

(f)

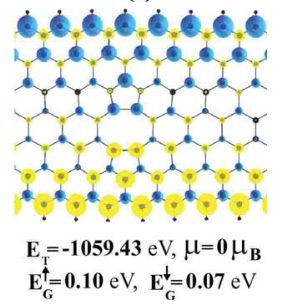

FIG. 4. (Color online) Vacancy and divacancy formations in an antiferromagnetic semiconductor ZGNR(14) with a repeat period of $l=8$. Carbon atoms at both edges are terminated by hydrogen atoms represented by small gray circles. Calculated total energy, $E_{T}$ (in eV/cell), net magnetic moment, $\mu$ (in Bohr magneton $\mu_{B} /$ cell), and band gap between spin-up(down) conduction and valence bands, $\left.E_{G}^{\uparrow(\downarrow)}\right)$ are shown for each case. Blue and yellow isosurfaces correspond to the difference of spin-up and spin-down states. [(a)-(c)] Single vacancy, [(d) and (e)] two separated single vacancy, and (f) a divacancy.

the works. As far as the effects of void on the band gap are concerned, the present discussion is unique. As we will show in Sec. III B, the situation is, however, rather different for zigzag nanoribbons.

\section{B. Zigzag nanoribbons}

Not only armchair but also zigzag nanoribbons are strongly affected by defects due to single and multiple vacancies. When coupled with the magnetic edge states of the zigzag nanoribbons, the vacancy defect brings about additional changes. The magnetic state and energy-band structure of these ribbons depend on the type and geometry of the defects. It should be noted that a $s p^{2}$-dangling bond is associated with each single vacancy in Figs. 4(a)-4(e). The effect of a defect generated from the single vacancy with a repeat periodicity of $l=8$ is examined in ZGNR $(14 ; 8)$ for three different positions. The total energy is $0.53 \mathrm{eV}$ lowered when the defect is situated at the edge rather than at the center of the ribbon.

$\operatorname{ZGNR}(14 ; 8)$ has a net magnetic moment of $\mu=1.94 \mu_{B}$ when the defect is situated at the center of the ribbon, and hence its antiferromagnetic ground state has changed to ferrimagnetic state through the magnetic moment of the single vacancy. Otherwise, $\mu$ becomes zero when the position of the defect deviates from the center. For example, in Figs. 4(b) and 4(c) the sum of the magnetic moments of the edge states is not zero, but the net magnetic moment per unit cell becomes zero only after the spins of the defect is added. Even if the net magnetic moment $\mu=0, \operatorname{ZGNR}(14 ; 8)$ does not have an antiferromagnetic ground state due to the presence of a single vacancy. The edge states, each normally having equal but opposite magnetic moments, become ferrimagnetic when a defect is introduced. The total magnetic moment of the supercell vanishes only after the magnetic moment of defect has been taken into account. Since the spin degeneracy has been broken, one can define $E_{G}^{\uparrow}$ and $E_{G}^{\downarrow}$ for majority and minority spin states. Not only the magnetic state but also the band gap of zigzag ribbons in Fig. 4 is affected by the symmetry and the position of the defect relative to the edges.

In Figs. 4(d) and 4(e) two defects associated with two separated vacancy exhibit similar behaviors. In Fig. 4(d) the vacancies sit in different sublattices and they have antiferromagnetic coupling with a zero net magnetic moment in the unit cell. On the other hand, the vacancies in Fig. 4(e) are located in the same sublattices and they are ferromagnetically coupled with a net magnetic moment of $3.91 \mu_{B}$. These results obtained for the zigzag nanoribbons appear to confirm the early tight-binding studies dealing with a $2 \mathrm{D}$ graphene layer. ${ }^{18,19,28,29,32}$ All carbon atoms surrounding the divacancy have threefold coordination, and hence there is no $s p^{2}$-dangling bond in the defect system. It appears that the relaxed structure is bipartite, since two missing atoms associated with the divacancy are situated in different sublattices and the calculated net magnetic moment per cell is zero.

According to Lieb's theorem the net magnetic moment per cell should be $\mu=1 \mu_{B}$ for the defect in Figs. 4(a) $-4(\mathrm{c})$ and $\mu=2 \mu_{B}$ for the case in Fig. 4(e). None of these cases is in agreement with Lieb's theorem. Here one can consider three features, which may be responsible from this discrepancy. First is the strong Jahn-Teller distortion and relaxation of carbon atoms at the close proximity of the defect. As a result some dangling $s p^{2}$ bonds reconstructed to form new $\mathrm{C}-\mathrm{C}$ covalent bonds. The lowering of the total energy, a driving force for such reconstruction, is as high as $0.5-0.6 \mathrm{eV} /$ cell. Second is the superexchange interaction with the magnetic edge states, which becomes effective for narrow ZGNRs. Third is the coexistence of unpaired $s p^{2}$-dangling bond and $\pi$ orbitals due to a missing atom. Our extensive analysis showed that the zigzag nanoribbons are rather different from armchair ones and these three features have a combined effect on the resulting net magnetic moment.

Finally, we note that introducing periodic vacancies or defects on a given ribbon appear to be difficult with the state-of-the-art technologies. Here we propose a method as described in Fig. 5 to achieve the formation of periodic local defects such as holes or vacancies. The sharp electrodes such as STM tips are situated at desired locations, such as one of the cases $1-3$ in Fig. 5(a) on the graphene ribbon with a given repeat periodicity. A potential difference, $V_{\text {tip }}$, common to all electrodes (tips) is applied between the tip and underlying insulator through the ribbon. This way the electronic potential of graphene atoms just below the tip is locally lowered or raised depending on the polarity of $V_{\text {tip }}$. Here, the effect of locally and periodically applied potential difference has been modeled by ETB, where the on-site energies of carbon atoms below the tip have been changed accordingly. Although the present model is crude, it still allows one to monitor the properties of nanoribbons. In Fig. 5(b) the varia- 


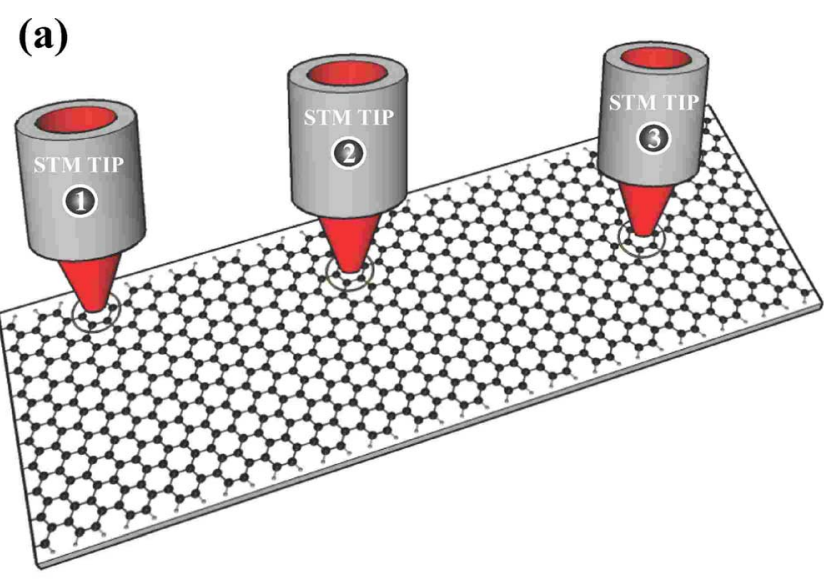

(b)

(c)

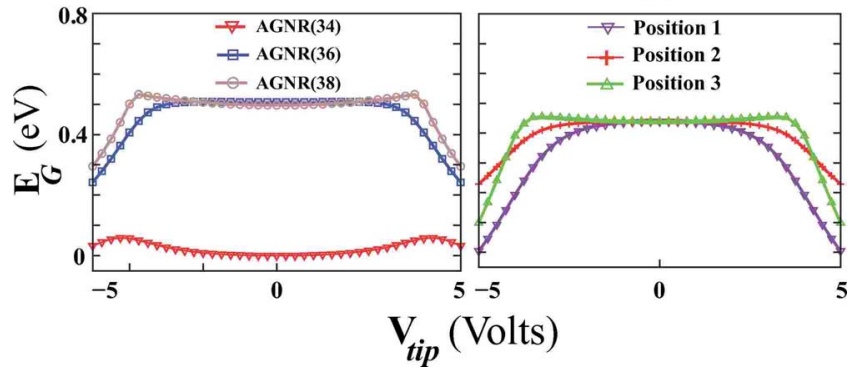

FIG. 5. (Color online) (a) Monitoring of band gaps $E_{G}$ by applying a local bias voltage $V_{\text {tip }}$ across the ribbon. (b) $E_{G}$ versus $V_{\text {tip }}$ applied at the center of $\operatorname{AGNR}(N ; 6)$ for $N=34,36$, and 38. (b) $E_{G}$ versus $V_{\text {tip }}$ of $\operatorname{AGNR}(34)$ for different tip positions schematically described at the top. The tip (or electrodes) is situated at one of the positions $1-3$. The repeat period is $l=20$.

tion in $E_{G}$ with $V_{\text {tip }}$ is calculated for $\operatorname{AGNR}(N ; 20)$ with $N$ $=34,36$, and 38 by using periodically located tips at the center of the ribbon. Because of the electron-hole symmetry in the ribbons, the band-gap variation depends on the magnitude of the bias voltage. In Fig. 5(c) the variation in the band gap of AGNR(42;20) with $V_{\text {tip }}$ and position of the tip are shown. In spite of the fact that the modifications of the band gaps are not the same as in Fig. 2, the available parameters, such as $V_{\text {tip }}, l$, tip geometry, and its position, make the monitoring of the properties possible.

\section{CONCLUSIONS}

In conclusion, we show that the energy band gaps and magnetic states of graphene nanoribbons can be modified by the periodic defects due to single or multiple vacancies. Itinerant (Bloch) states, which are perturbed by periodic vacancy defects, form dispersive bands at band edges and modify the band gap. The reconstruction of the atomic structure at the defect site is crucial for induced magnetic moments. The structural local reconstruction and spin polarization of the orbitals at the close proximity of the defect give rise to net magnetic moments, which, in turn, changes the magnetic ground state of the defect-free ribbon. The effect of the same vacancy defect on the electronic and magnetic properties depends on its symmetry, repeating periodicity, and position with respect to the edge of the ribbon, and it also depends on the type of the ribbon. While a nonmagnetic and semiconducting armchair nanoribbon can be metallized by divacancy, it becomes ferromagnetic by a single vacancy defect. The zigzag nanoribbon, which is an antiferromagnetic semiconductor, may become a ferrimagnetic semiconductor. However, the effect of vacancy defect on the electronic and magnetic properties of the zigzag nanoribbon varies with its symmetry, size, and position. Finally, we showed that electric field applied by an electrode perpendicular to the surface of the ribbon can induce effects similar to those due to vacancy defects.

\section{ACKNOWLEDGMENT}

Parts of the computations have been carried out by using UYBHM at Istanbul Technical University through Grant No. 2-024-2007.

\footnotetext{
*ciraci@fen.bilkent.edu.tr

${ }^{1}$ K. S. Novoselov, A. K. Geim, S. V. Morozov, D. Jiang, M. I. Katsnelson, I. V. Grigorieva, S. V. Dubonos, and A. A. Firsov, Nature (London) 438, 197 (2005).

${ }^{2}$ Y. Zhang, Y.-W. Tan, H. L. Stormer, and P. Kim, Nature (London) 438, 201 (2005).

${ }^{3}$ C. Berger, Z. Song, X. Li, X. Wu, N. Brown, C. Naud, D. Mayou, T. Li, J. Hass, A. N. Marchenkov, E. H. Conrad, P. N. First, and W. A. de Heer, Science 312, 1191 (2006).

${ }^{4}$ M. I. Katsnelson, K. S. Novoselov, and A. K. Geim, Nat. Phys. 2, 620 (2006).

${ }^{5}$ A. K. Geim and K. S. Novoselov, Nature Mater. 6, 183 (2007).

${ }^{6}$ M. Fujita, K. Wakabayashi, K. Nakada, and K. Kusakabe, J. Phys. Soc. Jpn. 65, 1920 (1996).

${ }^{7}$ K. Wakabayashi and M. Sigrist, Phys. Rev. Lett. 84, 3390 (2000).
}

${ }^{8}$ K. Wakabayashi, Phys. Rev. B 64, 125428 (2001).

${ }^{9}$ K. Wakabayashi, J. Phys. Soc. Jpn. 71, 2500 (2002).

${ }^{10}$ V. Barone, O. Hod, and G. E. Scuseria, Nano Lett. 6, 2748 (2006).

${ }^{11}$ Y.-W. Son, M. L. Cohen, and S. G. Louie, Nature (London) 444, 347 (2006).

${ }^{12}$ Y.-W. Son, M. L. Cohen, and S. G. Louie, Phys. Rev. Lett. 97, 216803 (2006).

${ }^{13}$ M. Y. Han, B. Ozyilmaz, Y. Zhang, and P. Kim, Phys. Rev. Lett. 98, 206805 (2007).

${ }^{14}$ K. Nordlund, J. Keinonen, and T. Mattila, Phys. Rev. Lett. 77, 699 (1996).

${ }^{15}$ A. Hashimoto, K. Suenaga, A. Gloter, K. Urita, and S. Iijima, Nature (London) 430, 870 (2004).

${ }^{16}$ P. Esquinazi, D. Spemann, R. Höhne, A. Setzer, K.-H. Han, and T. Butz, Phys. Rev. Lett. 91, 227201 (2003). 
${ }^{17}$ O. V. Yazyev and L. Helm, Phys. Rev. B 75, 125408 (2007).

${ }^{18}$ L. Brey, H. A. Fertig, and S. Das Sarma, Phys. Rev. Lett. 99, 116802 (2007).

${ }^{19}$ M. A. H. Vozmediano, M. P. Lopez-Sancho, T. Stauber, and F. Guinea, Phys. Rev. B 72, 155121 (2005).

${ }^{20}$ J. J. Palacios, J. Fernández-Rossier, and L. Brey, Phys. Rev. B 77, 195428 (2008); for graphene nanoislands see also J. Fernández-Rossier and J. J. Palacios, Phys. Rev. Lett. 99, 177204 (2007).

${ }^{21}$ X. Li, L. Zhang, S. Lee, and H. Dai, Science 319, 1229 (2008).

${ }^{22}$ X. Wang, Y. Ouyang, X. Li, H. Wang, J. Guo, and H. Dai, Phys. Rev. Lett. 100, 206803 (2008).

${ }^{23}$ P. E. Blochl, Phys. Rev. B 50, 17953 (1994).

${ }^{24}$ J. P. Perdew, J. A. Chevary, S. H. Vosko, K. A. Jackson, M. R. Pederson, D. J. Singh, and C. Fiolhais, Phys. Rev. B 46, 6671 (1992).

${ }^{25}$ G. Kresse and J. Hafner, Phys. Rev. B 47, 558 (1993).

${ }^{26}$ G. Kresse and J. Furthmüller, Phys. Rev. B 54, 11169 (1996).

${ }^{27}$ As shown by the recent work based on self-energy corrections
[L. Yang, C. H. Park, Y.-W. Son, M. L. Cohen, and S. G. Louie, Phys. Rev. Lett. 99, 186801 (2007)], the band gap, $E_{G}$, is underestimated by the DFT calculations. Since we consider structures which have already a band gap, its actual value does not affect our discussion in any essential manner.

${ }^{28}$ Vitor M. Pereira, F. Guinea, J. M. B. Lopes dos Santos, N. M. R. Peres, and A. H. Castro Neto, Phys. Rev. Lett. 96, 036801 (2006).

${ }^{29}$ N. M. R. Peres, F. Guinea, and A. H. Castro Neto, Phys. Rev. B 73, 125411 (2006).

${ }^{30}$ Using the definition of Schottky defect, we calculated the formation energy of relaxed vacancy of single carbon atom in AGNR(34) to be $7.79 \mathrm{eV}$. This value is smaller than the binding energy of a carbon atom in the graphene, which is calculated to be 9.23 . However, owing to the concerted process the vacancy formation energy may be smaller than $7.79 \mathrm{eV}$.

${ }^{31}$ E. H. Lieb, Phys. Rev. Lett. 62, 1201 (1989).

${ }^{32}$ H. Kumazaki and Dai S. Hirashi, J. Phys. Soc. Jpn. 76, 064713 (2007). 